\title{
Rapid whole genome optical mapping of Plasmodium falciparum
}

\author{
Matthew C Riley ${ }^{1 *}$, Benjamin C Kirkup Jr ${ }^{2,4}$, Jake D Johnson ${ }^{3}$, Emil P Lesho ${ }^{2,4}$ and Christian F Ockenhouse ${ }^{1}$
}

\begin{abstract}
Background: Immune evasion and drug resistance in malaria have been linked to chromosomal recombination and gene copy number variation (CNV). These events are ideally studied using comparative genomic analyses; however in malaria these analyses are not as common or thorough as in other infectious diseases, partly due to the difficulty in sequencing and assembling complete genome drafts. Recently, whole genome optical mapping has gained wide use in support of genomic sequence assembly and comparison. Here, a rapid technique for producing whole genome optical maps of Plasmodium falciparum is described and the results of mapping four genomes are presented.

Methods: Four laboratory strains of $P$. falciparum were analysed using the Argus ${ }^{\mathrm{TM}}$ optical mapping system to produce ordered restriction fragment maps of all 14 chromosomes in each genome. Plasmodium falciparum DNA was isolated directly from blood culture, visualized using the Argus ${ }^{\mathrm{TM}}$ system and assembled in a manner analogous to next generation sequence assembly into maps (AssemblyViewerTM, OpGen Inc. ${ }^{\circledR}$ ). Full coverage maps were generated for $P$. falciparum strains 3D7, FVO, D6 and C235. A reference $P$. falciparum in silico map was created by the digestion of the genomic sequence of $P$. falciparum with the restriction enzyme Aflll, for comparisons to genomic optical maps. Maps were then compared using the MapSolver ${ }^{\mathrm{TM}}$ software.
\end{abstract}

Results: Genomic variation was observed among the mapped strains, as well as between the map of the reference strain and the map derived from the putative sequence of that same strain. Duplications, deletions, insertions, inversions and misassemblies of sizes ranging from 3,500 base pairs up to 78,000 base pairs were observed. Many genomic events occurred in areas of known repetitive sequence or high copy number genes, including var gene clusters and rifin complexes.

Conclusions: This technique for optical mapping of multiple malaria genomes allows for whole genome comparison of multiple strains and can assist in identifying genetic variation and sequence contig assembly. New protocols and technology allowed us to produce high quality contigs spanning four P. falciparum genomes in six weeks for less than $\$ 1,000.00$ per genome. This relatively low cost and quick turnaround makes the technique valuable compared to other genomic sequencing technologies for studying genetic variation in malaria.

\section{Background}

Malaria is caused by various species of the genus Plasmodium, the most prevalent and deadly of which is Plasmodium falciparum[1,2]. With $\sim 40 \%$ of the world's population at risk for malaria, efforts in prevention, eradication and treatment of the disease are globally vital $[2,3]$. Despite efforts to develop vaccines and drugs to combat malaria, vaccine escape and drug resistance

\footnotetext{
* Correspondence: matthew.curtis.riley@us.army.mil

'Walter Reed Army Institute of Research, Division of Malaria Vaccine

Development, Silver Spring, Maryland, USA

Full list of author information is available at the end of the article
}

continue to be a problem $[1,4,5]$. The ability to compare whole genomes could assist in these efforts, as genetic variation and recombination have been shown to facilitate antigen diversity, immune escape and evolution of anti-malarial drug resistance [4,6-9]. Proposed mechanisms for these events include chromosome translocation and recombination, segmental duplication and $\mathrm{CNV}$ [10-13]. Ideally, fully sequenced and assembled genomes would be a way to study these mechanisms; however, this continues to be expensive and labour intensive [12-14]. Optical mapping provides an alternative to study these events, but to date has not been used for

\section{Biomed Central}

(c) 2011 Riley et al; licensee BioMed Central Ltd. This is an Open Access article distributed under the terms of the Creative Commons Attribution License (http://creativecommons.org/licenses/by/2.0), which permits unrestricted use, distribution, and reproduction in any medium, provided the original work is properly cited. 
this purpose in malaria, partly due to the impracticality of scaling up previous mapping techniques to multiple genomes [15].

Optical mapping is a technique during which visualization of high molecular weight DNA cut by restriction enzymes is used to create a scaffold fragment pattern across a genome (Figure 1). The first and most critical step in this process is the purification of extremely high molecular weight DNA (150Kb-1.6Mb). This was accomplished in this case by using magnetic bead technology. The DNA is then bound to a glass surface and subjected to a pre-determined restriction enzyme and labelled using a fluorescent dye. These digested, bound molecules are then imaged and relative sizes are assigned to generate a cut site pattern. These patterns are used to assemble the molecules against each other, generating a digital representation of fragment pattern alignments, or an "optical map"[16].

The 14 chromosome, $14 \mathrm{Mbp}$ P. falciparum strain 3D7 genome was published in 2002, and to date this is the only complete, assembled genome sequence for this organism [14]. Optical mapping of DNA was used to assist in assembly of this published genome sequence, but until now has not been widely used on any other species or strains $[15,17]$. The Argus ${ }^{\mathrm{TM}}$ optical mapping system represents a radical advancement in whole genome optical mapping technology, and is being widely used for studies in bacterial identification, evolution, comparative genomics and

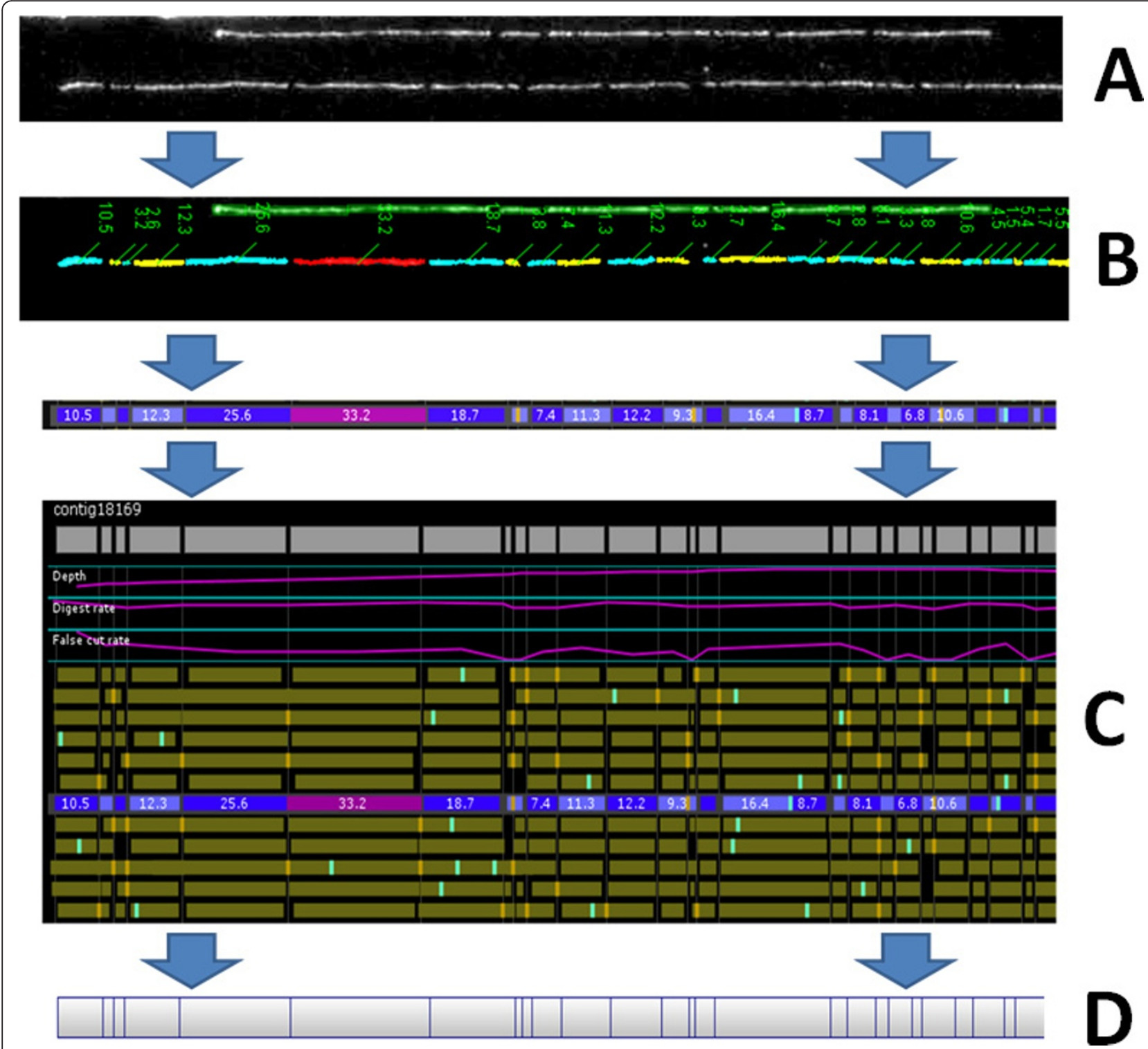

Figure 1 Generating an Optical Map; After DNA is bound and digested, imaging of molecules is performed (A). The fragments are assigned sizes (B) and assembled (C) to generate a consensus optical map (D). Imaging, cut site and DNA length prediction is automatic, and represented here as spaces between the colored, labeled fragments. 
genome assembly [18-22]. By developing new parasite isolation techniques and DNA extraction protocols, stated Argus $^{\mathrm{TM}}$ limitations were surpassed to produce whole genome maps of malaria genomes for under $\$ 1,000.00$ each, fully assembled in less than one week.

\section{Results}

\section{Parasite isolation and DNA extraction}

Two vaccine research $P$. falciparum strains and two drug resistance research strains were selected for this work. Strains 3D7 and FVO are commonly used in vaccine development and 3D7 allowed the direct comparison of its optical map and the sequence based in silico map which was used as a reference [14]. Strains D6 (NC_004317) and C235 were selected based on their opposing anti-malarial drug resistance profiles; D6 is generally considered drug sensitive with minor mefloquine resistance, while C235 is considered a multi-drug resistant strain [Unpublished Data]. All four parasites are culture-adapted strains and were grown to 4-6\% parasitaemia in $6 \mathrm{~mL}$ standard blood culture prior to purification. Isolation of late ring- early schizont-stage parasites was performed by multiple $4^{\circ} \mathrm{C}$ wash/centrifuge cycles after initial lysis with $0.1 \%$ saponin in PBS. The blood culture was centrifuged at 2,486 rpm (860 rcf) and $4^{\circ} \mathrm{C}$ for 6 minutes and the pellet resuspended in one-half starting volume of ice cold $0.1 \%$ saponin/PBS $(\mathrm{w} / \mathrm{v})$. This solution was allowed to incubate on ice for 10 minutes and then centrifuged again at 2,486 rpm (860 rcf) and $4^{\circ} \mathrm{C}$ for 6 minutes. After removal of supernatant, the pellet was resuspended in ice cold DNAse free water and centrifuged again at 2,486 rpm (860 rcf) and $4^{\circ} \mathrm{C}$ for 6 minutes. This protocol resulted in release of malaria parasites and elimination of RBCs (Figure 2). This pellet was resuspended in $50 \mu \mathrm{L}$ of ice cold DNAse free water and used directly in DNA extraction.

DNA extraction was performed on this purified parasite sample according to the Gram-negative Bacterial DNA Preparation protocol available from Opgen, Inc. ${ }^{\circledR}$. The parasites were lysed and resulting DNA was mixed with magnetic beads, subjected to a variety of wash buffers and eluted off the beads using wide bore pipette tips. Modifications to the manufacturer's protocol included addition of a 20 minute extension of initial lysis time, reduction in elution volume from $90 \mu \mathrm{L}$ to $75 \mu \mathrm{L}$, and an extra two elutions at $65^{\circ} \mathrm{C}$ for 15 minutes each. Between $4 \mu \mathrm{L}$ and $6 \mu \mathrm{L}$ DNA was applied to mapcards (cards run on the Argus ${ }^{\mathrm{TM}}$ ) and run on the Argus ${ }^{\mathrm{TM}}$ system per manufacturer's specifications, after proper quality controls checks and appropriate dilutions using Opgen ${ }^{\mathbb{R}}$ QCards.

\section{Mapset filter and contig assembly}

Contig assembly was performed using the Argus ${ }^{\mathrm{TM}}$ software, but with changes to default settings. The mapset (total dataset generated from a single run) was filtered for minimum molecule (chromosome segment imaged) size $(>150 \mathrm{~Kb})$, minimum fragments per molecule $(>12)$ and minimum molecule quality $(>0.4)$. Each genome was able to be assembled on as few as two mapcards; however, in some cases more cards were used in an attempt to resolve difficult telomeric chromosome ends. The data from each additional run on the same strain were combined together for final assembly. Assembly was conducted using either the "fastest" setting or "slower" setting, with removal of default circularization parameters. Partial assembly results were saved when 14 contigs became apparent by having $>50$ molecules each. Contigs were split off and reassembled against the original mapset individually using the "Find Hits" feature. Contigs were considered "finished" when no additional molecules were added by subsequent reassemblies. The only areas that did not pass default Argus ${ }^{\mathrm{TM}}$ quality control (QC) settings were telomeric repeats near some chromosome ends. Chromosome ends that were not blunt were visually inspected and any questionable molecule was removed from the final map. Finished contigs were imported and aligned using Opgen ${ }^{\circledR}$ MapSolver ${ }^{\text {TM }}$ software with default alignment settings (Figure 3).

\section{Genome alignment and comparative genomics}

Each genome was aligned against the 3D7 reference genome and genomic differences were noted and compared against the other strains (Table 1). Relative to the 3D7 reference DNA sequence, the 3D7 optical map showed two probable misassemblies, one gene duplication and two insertion/deletion events. Overall, this accounts for $\sim 304 \mathrm{~Kb}$ of unaligned sequence. The multi-drug resistant strain $\mathrm{C} 235$ shows a $\sim 70 \mathrm{~Kb}$ insertion that is of particular interest as it is the largest observed difference and is completely unique to this genome (Figure 4).

Each genomic event was aligned to the reference sequence from PlasmoDB [23], and the DNA sequence for the insertion (or flanking DNA for deletions) was referenced to associated genes. In many cases var or rifin complexes were associated with these events, in particular PfEMP1, a known source of antigenic variation [24]. One identified var cluster of known variation on chromosome 4 is shown in Figure 5 in the four genomes mapped aligned to the reference genome.

\section{Discussion}

The major hurdle to optically mapping P. falciparum was sample preparation. Because the parasites can be grown only in blood culture, there is a possibility of contamination of the sample by human white blood cells or nucleated erythrocytes. As there is no 


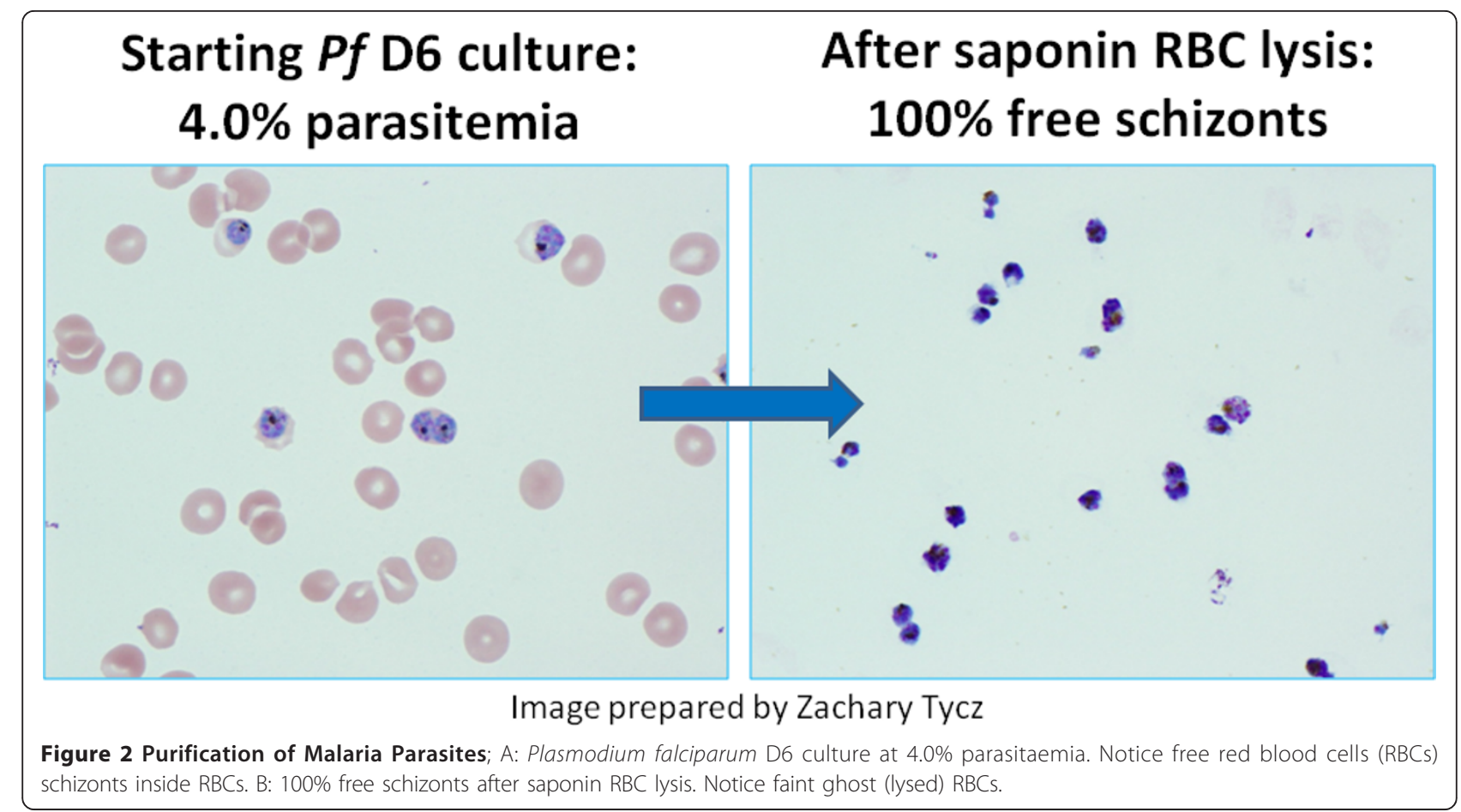

amplification involved in this technique, even a single human cell (3,300 Mb DNA/cell) could overwhelm the sample with human DNA and mask the comparatively small $24 \mathrm{Mb} /$ cell malaria genome. This was overcome using the parasite isolation technique described in the methods. The success of this method was such that no contigs in the assemblies failed to match a region of the sequenced Plasmodium genome.

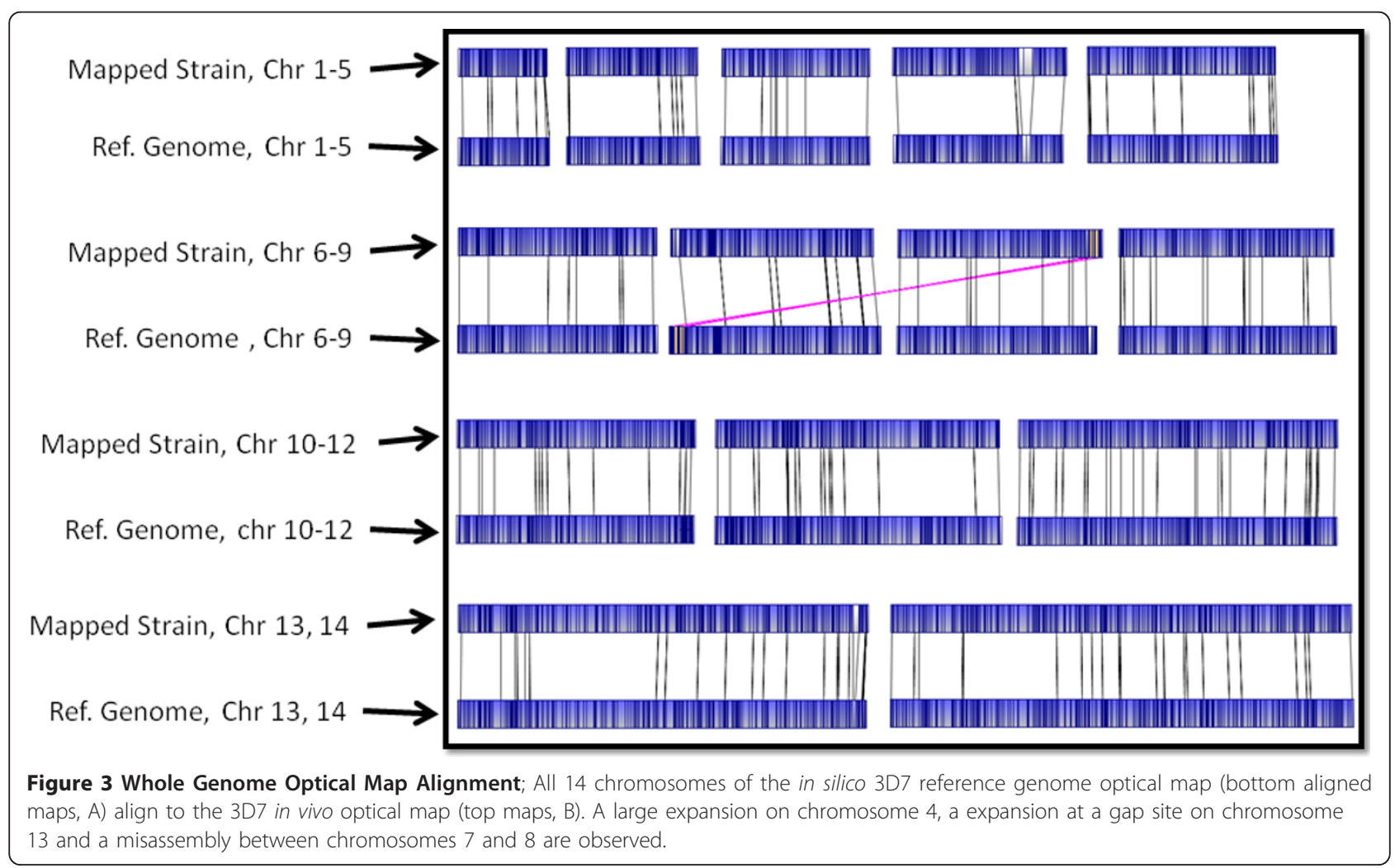


Table 1 Summary of Results

\begin{tabular}{ccccc}
\hline Genome & Total Length $(\mathbf{M b})$ & Geographic Area & Variations to Reference $^{\mathbf{a}}$ & Maximum Single Variation $^{\mathbf{b}}$ \\
\hline 3D7 Reference & 23.264 & West Africa & $\mathrm{N} / \mathrm{A}$ & $\mathrm{N} / \mathrm{A}$ \\
\hline 3D7 Optical Map & 23.165 & West Africa & 4 & $46.0 \mathrm{~Kb}$ \\
\hline D6 & 21.958 & East Africa & 5 & $43.6 \mathrm{~Kb}$ \\
\hline FVO & 22.158 & Southeast Asia & 4 & $41.4 \mathrm{~Kb}$ \\
\hline C235 & 21.734 & Southeast Asia & 5 & $77.5 \mathrm{~Kb}$ \\
\hline
\end{tabular}

The total megabases $(\mathrm{Mb})$ of each genome predicted from alignment against the in silico optical map (or reference), the geographic area each strain was isolated from, the number of variations from each map to the reference genome and the largest (in Kb) observed difference. Variation under $2 \mathrm{~Kb}$ and telomeric DNA were not included.

${ }^{a}$ represents independent differences observed against the reference genome using default assembly parameters. Results can vary by settings.

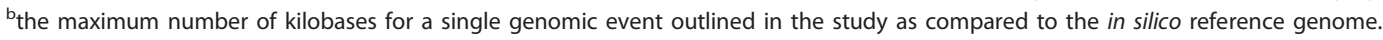
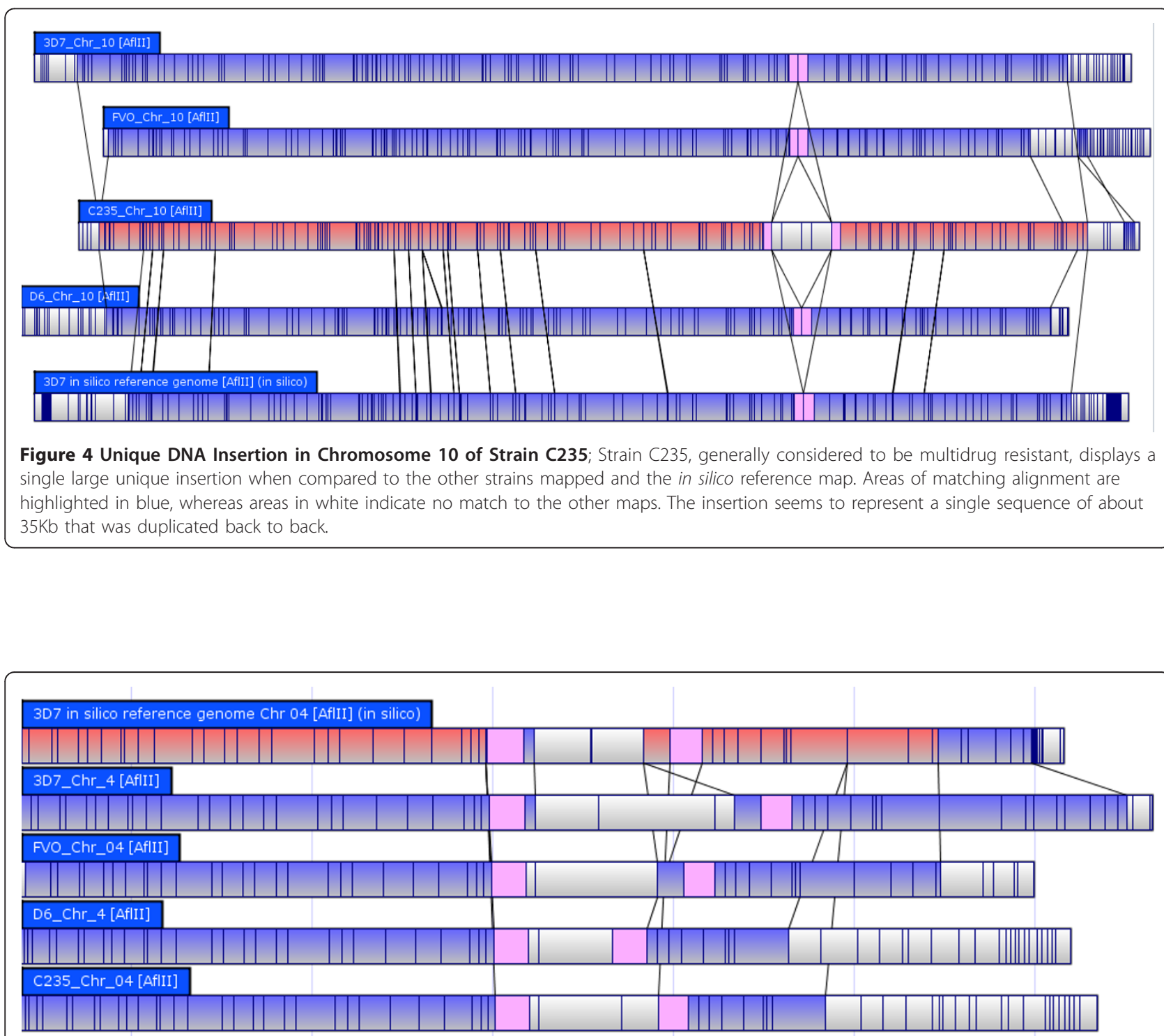

Figure 5 Chromosome 4 Region of Variation in all Strains; Compared to the reference genome, all strains mapped generally align (in blue) to the reference genome (red). However, the white area indicates no alignment in any genome to another, indicating a high degree of variation. 
Some chromosome ends did not readily align to the reference strain. These unaligned map ends often had poor QC scores and low coverage. Using AflII as a mapping enzyme would result in multiple small AflII fragments at the end containing telomeric repeats which would be not resolved. Thus, while there may be more DNA at the ends of some chromosomes than is represented in our optical maps, the DNA and related cut site patterns internal to these ends are correct. Blunt ends were observed at some contig ends and passed QC indicating that we resolved the full length of the chromosome (Figure 6).

Complete chromosome end translocations were observed in C235 and FVO, indicating this could be a frequently observed difference between distantly related strains. A single region on chromosome 4 varied significantly in length (up to $65 \mathrm{~Kb}$ ) among the strains studied. It includes a known var gene cluster in the reference genome (Figure 7) [6]. The var clusters (which contain variants of PfEMP1) are known to be associated with antigenic variation and are thought to be a candidate for vaccine development $[6,9,24]$. In the 3D7 optical map, an exact duplication of PfEMP1 was observed in comparison to other strains and the reference genome (Figure 8). PfEMP1 is a known source of antigenic variation, and while segmental duplication has been implicated as a possible mechanism, until now it has not been directly observed [24]. Strain C235 is considered multi-drug resistant and thus any unique genomic events compared to susceptible strains are of particular interest [Unpublished Data]. While there were several non-unique insertions and deletions compared to the reference genome, we did observe a single $70 \mathrm{~Kb}$ insertion on C235 chromosome 10 that does not have a similar pattern in any of the other genomes mapped (Figure 4). This insert seems to be a $35 \mathrm{~Kb}$ segment of DNA that was duplicated back to back, which is potentially meaningful as drug resistance is often associated with gene duplication and CNV [4]. Using Mapsolver ${ }^{\mathrm{TM}}$ UPGMA phylogenetic trees for individual chromosomes, 3D7 was found to be most similar to D6 (9/14 chromosomes), while C235 and FVO were most closely related ( $9 / 14$ chromosomes). These relationships most likely reflect the geographic regions that each parasite is from (Table 1).

\section{Conclusions}

Optical genomic mapping technology represents a significant advancement in comparative genomic research of malaria parasites. The optical maps of the four P. falciparum strains presented here demonstrate significant genome variation, much of which can be traced to regions known to contain coding sequence implicated in antigenic variation. This confirms findings generated via more difficult and expensive research methods that have suggested

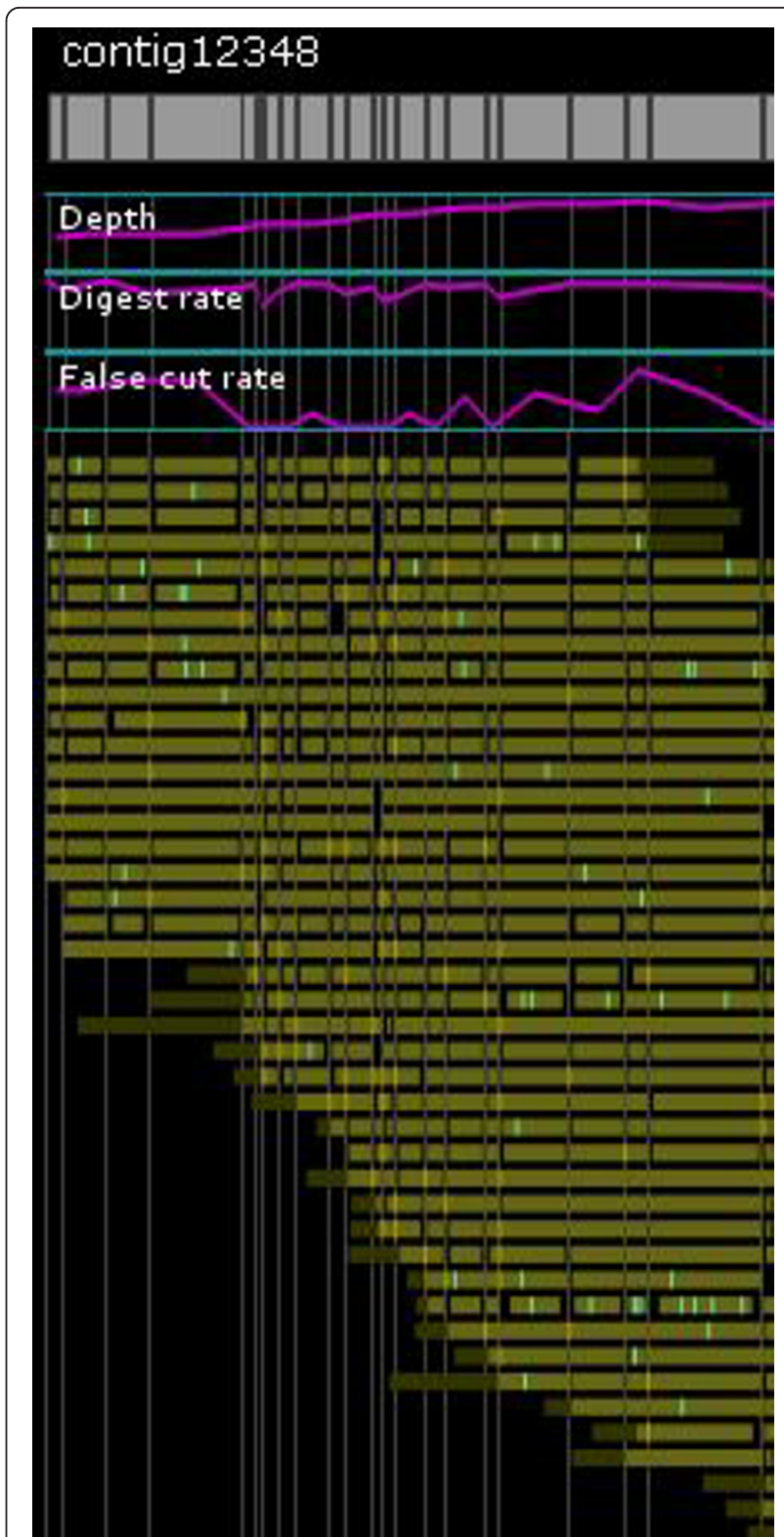

Figure 6 Optical Resolution of Blunt Chromosome Ends; Unlike bacterial optical maps, malaria DNA is not circular. Telomeric repeats near the ends of some chromosomes can cause the map to be truncated, unless a "blunt" end is observed as shown in this figure. "Blunt" ends in optical mapping refer not to enzyme cut sites but the true end of a chromosome as depicted by having all individual molecules "end" at the same sequence.

chromosomal translocations and segmental duplications are associated with immune escape and drug resistance in malaria [6-8,10,11]. Optical mapping resolves these types of genomic events and the techniques described in this paper allow for inexpensive, rapid, robust production of whole genome optical maps for malaria. This method also opens the door for assistance with genome assembly, as has been demonstrated with optical mapping of other 


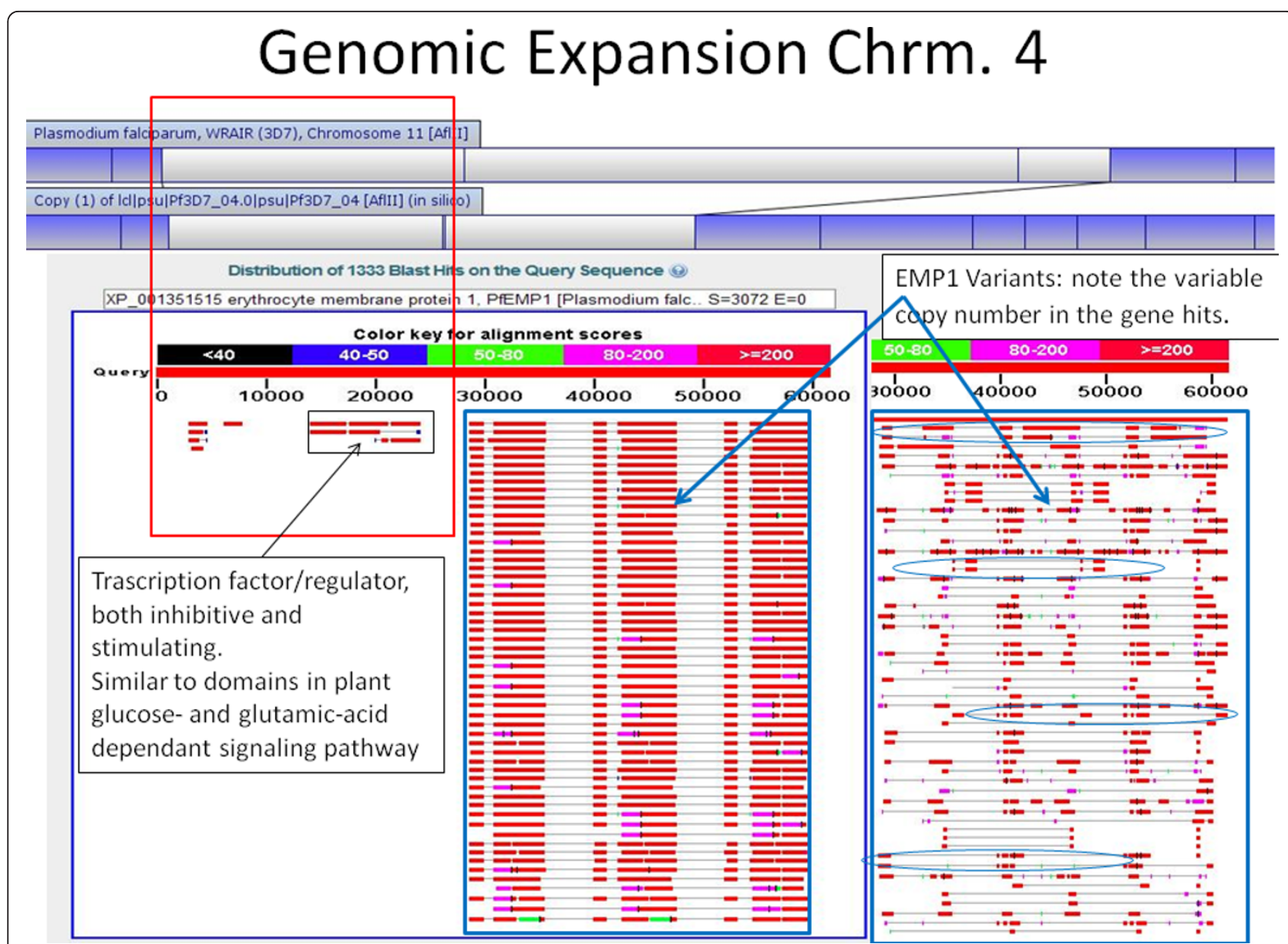

Figure 7 Chromosome 4 Variable Region Associated with var Cluster; The variable region on chromosome 4 shown in Figure 6 contains DNA that matches to an area of known variation. Here, you can see the BLAST result of the repetitive sequence found in this region. [http:// blast.ncbi.nlm.nih.gov/Blast.cgi]

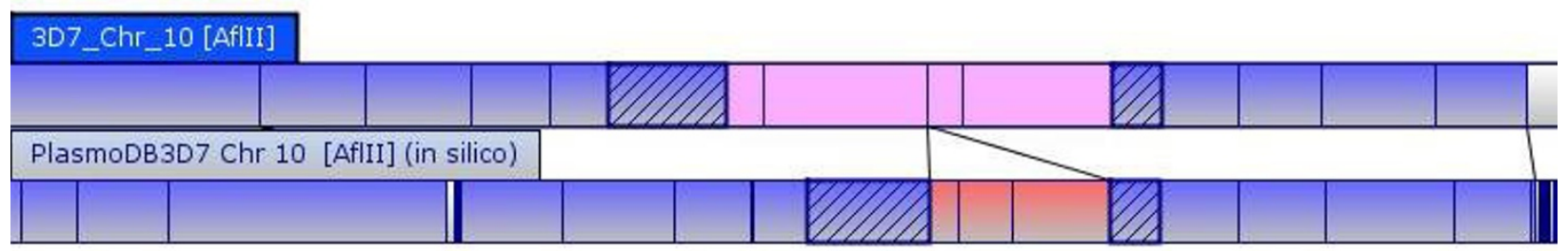

Figure 8 Chromosome 10 Duplication of PfEMP1; The segmental duplication observed in Figure 3 is located on chromosome 10 and is potentially an exact duplication of the gene PFEMP1.

large genomes [25-27]. The development of optical maps and fully assembled malaria genomes will increase knowledge of genetic variation in malaria parasites and thus enhance the ability to combat this disease.

\section{Acknowledgements}

We would like to thank Kathy Moch and Patty Lee for parasite culture, Charlotte Lanteri and Zachary Tycz for development and assistance with parasite isolation techniques, Edwin Kamau for advice on DNA preparation and Opgen ${ }^{\circledR}$, Inc. for technical support with special thanks to Trevor Wagner for advice and assistance with DNA preparation and software manipulation and Louis Gardner and Emily Zentz for assistance with sequence assembly. Disclaimer

The opinions or assertions contained herein are the private views of the authors, and are not to be construed as official, or as reflecting true views of the Department of the Army or the Department of Defense.

\section{Author details}

'Walter Reed Army Institute of Research, Division of Malaria Vaccine Development, Silver Spring, Maryland, USA. Walter Reed Army Institute of 
Research, Division of Bacterial and Rickettsial Diseases, Silver Spring Maryland, USA. ${ }^{3}$ Walter Reed Army Institute of Research, Division of Experimental Therapeutics, Silver Spring, Maryland, USA. ${ }^{4}$ Uniformed Services University of the Health Sciences, Silver Spring, Maryland, USA.

\section{Authors' contributions}

MCR performed parasite isolation and DNA purification, Optical mapping, data analysis and drafted the manuscript. BCK helped conceive the study, participated in its design and coordination and helped draft the manuscript. JJ assisted with parasite isolation, provided strains C235 and D6, characterized their drug resistance and helped draft the manuscript. EL participated in the design and coordination of the study and provided training and support for the optical mapping. CO helped conceive of the study, directed the study design and provided strains 3D7 and FVO. All authors read and approved the final manuscript.

\section{Competing interests}

The authors declare that they have no competing interests.

Received: 1 June 2011 Accepted: 26 August 2011

Published: 26 August 2011

\section{References}

1. Hay SI, Okiro EA, Gething PW, Patil AP, Tatem AJ, Guerra CA, Snow RW: Estimating the global clinical burden of Plasmodium falciparum malaria in 2007. PLoS Med 2010, 7:e1000290.

2. Snow RW, Guerra CA, Noor AM, Myint HY, Hay Sl: The global distribution of clinical episodes of Plasmodium falciparum malaria. Nature 2005, 434:214-217.

3. World Health Organization: World Malaria Report 2010 Switzerland: World Health Organization Press; 2010.

4. Imwong M, Dondorp AM, Nosten F, Yi P, Mungthin M, Hanchana S, Das D, Phyo AP, Lwin KM, Pukrittayakamee S, Lee SJ, Saisung S, Koecharoen K, Nguon C, Day NP, Socheat D, White NJ: Exploring the contribution of candidate genes to artemisinin resistance in Plasmodium falciparum. Antimicrob Agents Chemother 2010, 54:2886-2892.

5. Cohen J, Nussenzweig V, Nussenzweig R, Vekemans J, Leach A: From the circumsporozoite protein to the RTS, S/AS candidate vaccine. Hum Vaccin 2010, 6:90-96.

6. Frank M, Kirkman L, Costantini D, Sanyal S, Lavazec C, Templeton TJ, Deitsch KW: Frequent recombination events generate diversity within the multi-copy variant antigen gene families of Plasmodium falciparum. Int J Parasitol 2008, 38:1099-1109.

7. Mackinnon MJ, Marsh K: The selection landscape of malaria parasites. Science 2010, 328:866-871.

8. Ferreira MU, da Silva Nunes M, Wunderlich G: Antigenic diversity and immune evasion by malaria parasites. Clin Diagn Lab Immunol 2004, 11:987-995.

9. Good MF, Doolan DL: Malaria vaccine design: immunological considerations. Immunity 2010, 33:555-566.

10. Zilversmit MM, Volkman SK, DePristo MA, Wirth DF, Awadalla P, Hartl DL Low-complexity regions in Plasmodium falciparum: missing links in the evolution of an extreme genome. Mol Biol Evol 2010, 27:2198-2209.

11. Kooij TW, Carlton JM, Bidwell SL, Hall N, Ramesar J, Janse CJ, Waters AP: A Plasmodium whole-genome synteny map: indels and synteny breakpoints as foci for species-specific genes. PLoS Pathog 2005, 1:e44.

12. $\mathrm{Mu} \mathrm{J}$, Seydel KB, Bates $\mathrm{A}$, Su XZ: Recent progress in functional genomic research in Plasmodium falciparum. Curr Genomics 2010, 11:279-286.

13. Samarakoon U, Regier A, Tan A, Desany BA, Collins B, Tan JC, Emrich SJ, Ferdig MT: High-throughput 454 resequencing for allele discovery and recombination mapping in Plasmodium falciparum. BMC Genomics 2011, 12:116.

14. Gardner MJ, Hall N, Fung E, White O, Berriman M, Hyman RW, Carlton JM, Pain A, Nelson KE, Bowman S, Paulsen IT, James K, Eisen JA, Rutherford K, Salzberg SL, Craig A, Kyes S, Chan MS, Nene V, Shallom SJ, Suh B, Peterson J, Angiuoli S, Pertea M, Allen J, Selengut J, Haft D, Mather MW, Vaidya AB, Martin DM, Fairlamb AH, Fraunholz MJ, Roos DS, Ralph SA, McFadden Gl, Cummings LM, Subramanian GM, Mungall C, Venter JC Carucci DJ, Hoffman SL, Newbold C, Davis RW, Fraser CM, Barrell B: Genome sequence of the human malaria parasite Plasmodium falciparum. Nature 2002, 419:498-511.
15. Jing J, Lai Z, Aston C, Lin J, Carucci DJ, Gardner MJ, Mishra B, Anantharaman TS, Tettelin H, Cummings LM, Hoffman SL, Venter JC, Schwartz DC: Optical mapping of Plasmodium falciparum chromosome 2. Genome Res 1999, 9:175-181.

16. Kotewicz ML, Mammel MK, LeClerc JE, Cebula TA: Optical mapping and 454 sequencing of Escherichia coli $0157: \mathrm{H7}$ isolates linked to the US 2006 spinach-associated outbreak. Microbiology 2008, 154(Pt 11):3518-28.

17. Lai Z, Jing J, Aston C, Clarke V, Apodaca J, Dimalanta ET, Carucci DJ, Gardner MJ, Mishra B, Anantharaman TS, Paxia S, Hoffman SL, Craig Venter J, Huff EJ, Schwartz DC: A shotgun optical map of the entire Plasmodium falciparum genome. Nat Genet 1999, 23:309-313.

18. Zhou S, Kile A, Bechner M, Place M, Kvikstad E, Deng W, Wei J, Severin J, Runnheim R, Churas C, Forrest D, Dimalanta ET, Lamers C, Burland V, Blattner FR, Schwartz DC: Single-molecule approach to bacterial genomic comparisons via optical mapping. J Bacteriol 2004, 186:7773-7782.

19. Giongo A, Tyler HL, Zipperer UN, Triplett EW: Two genome sequences of the same bacterial strain, Gluconacetobacter diazotrophicus PAI 5, suggest a new standard in genome sequence submission. Stand Genomic Sci 2010, 2:309-317.

20. Latreille P, Norton S, Goldman BS, Henkhaus J, Miller N, Barbazuk B, Bode HB, Darby C, Du Z, Forst S, Gaudriault S, Goodner B, Goodrich-Blair H, Slater S: Optical mapping as a routine tool for bacterial genome sequence finishing. BMC Genomics 2007, 8:321.

21. Saunders MP, Wu G, Abuoun M, Pan Z, Anjum M, Woodward MJ: Optical genetic mapping defines regions of chromosomal variation in serovars of S. enteric subsp. enterica of concern for human and animal health. Epidemiol Infect 2010, 22:1-10.

22. Johnson PD, Ballard SA, Grabsch EA, Stinear TP, Seemann T, Young HL, Grayson ML, Howden BP: A sustained hospital outbreak of vancomycinresistant Enterococcus faecium bacteremia due to emergence of vanB E. faecium sequence type 203. J Infect Dis 2010, 202:1278-1286.

23. Aurrecoechea C, Brestelli J, Brunk BP, Dommer J, Fischer S, Gajria B, Gao X, Gingle A, Grant G, Harb OS, Heiges M, Innamorato F, lodice J, Kissinger JC, Kraemer E, Li W, Miller JA, Nayak V, Pennington C, Pinney DF, Roos DS, Ross C, Stoeckert CJ Jr, Treatman C, Wang H: PlasmoDB: a functional genomic database for malaria parasites. Nucleic Acids Res 2009, 37(Database issue):D539-43[http://plasmodb.org/plasmo/].

24. Pasternak ND, Dzikowski R: PfEMP1: an antigen that plays a key role in the pathogenicity and immune evasion of the malaria parasite Plasmodium falciparum. Int I Biochem Cell Biol 2009, 41:1463-1466.

25. Zhou S, Kile A, Kvikstad E, Bechner M, Severin J, Forrest D, Runnheim R, Churas C, Anantharaman TS, Myler P, Vogt C, Ivens A, Stuart K, Schwartz DC: Shotgun optical mapping of the entire Leishmania major Friedlin genome. Mol Biochem Parasitol 2004, 138:97-106.

26. Zhou S, Wei F, Nguyen J, Bechner M, Potamousis K, Goldstein S, Pape L, Mehan MR, Churas C, Pasternak S, Forrest DK, Wise R, Ware D, Wing RA, Waterman MS, Livny M, Schwartz DC: A single molecule scaffold for the maize genome. PLoS Genet 2009, 5:e1000711.

27. Teague B, Waterman MS, Goldstein S, Potamousis K, Zhou S, Reslewic S, Sarkar D, Valouev A, Churas C, Kidd JM, Kohn S, Runnheim R, Lamers C, Forrest D, Newton MA, Eichler EE, Kent-First M, Surti U, Livny M, Schwartz DC: High-resolution human genome structure by singlemolecule analysis. Proc Natl Acad Sci USA 2010, 107:10848-10853.

\section{doi:10.1186/1475-2875-10-252}

Cite this article as: Riley et al.: Rapid whole genome optical mapping of Plasmodium falciparum. Malaria Journal 2011 10:252. 